Reprinted from

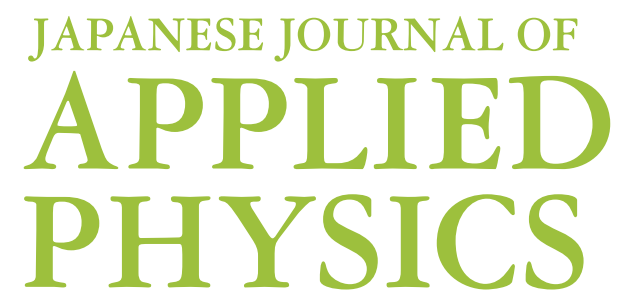

REGULAR PAPER

Experimental Observation of Enhanced Electron-Phonon Interaction in Suspended Si Double Quantum Dots

Jun Ogi, Thierry Ferrus, Tetsuo Kodera, Yoshishige Tsuchiya, Ken Uchida,

David A. Williams, Shunri Oda, and Hiroshi Mizuta

Jpn. J. Appl. Phys. 49 (2010) 045203

(C) 2010 The Japan Society of Applied Physics 


\title{
Experimental Observation of Enhanced Electron-Phonon Interaction in Suspended Si Double Quantum Dots
}

\author{
Jun Ogi1 ${ }^{1,5 *}$, Thierry Ferrus ${ }^{2}$, Tetsuo Kodera ${ }^{1}$, Yoshishige Tsuchiya ${ }^{3,5}$, Ken Uchida ${ }^{4,5}$, \\ David A. Williams ${ }^{2}$, Shunri Oda ${ }^{1,5}$, and Hiroshi Mizuta ${ }^{3,4,5}$ \\ ${ }^{1}$ Quantum Nanoelectronics Research Center, Tokyo Institute of Technology, Meguro, Tokyo 152-8552, Japan \\ ${ }^{2}$ Hitachi Cambridge Laboratory, Cambridge, Cambridgeshire CB3 OHE, U.K. \\ ${ }^{3}$ School of Electronics and Computer Science, University of Southampton, Southampton, Hampshire SO17 1BJ, U.K. \\ ${ }^{4}$ Department of Physical Electronics, Tokyo Institute of Technology, Meguro, Tokyo 152-8552, Japan \\ ${ }^{5}$ SORST-JST (Japan Science and Technology Agency), 4-1-8 Honcho, Kawaguchi, Saitama 332-0012, Japan \\ Received December 4, 2009; accepted January 18, 2010; published online April 20, 2010
}

Silicon-based suspended double quantum dots (SDQDs) were fabricated to study and control the strength of the electron-phonon interaction. A distinctive and large inelastic tunneling was observed in single-electron transport measurement and well explained by the emission of phonons that interact strongly with electrons owing to the phonon modulation in the suspended film. The first time observation of the enhancement of the electron-phonon interaction in Si SDQDs as well as the good agreement between the experimental results and the theoretical simulations are encouraging preliminary results that allow us to envision the observation of the tailoring of the electron-phonon interaction in SDQDs.

(C) 2010 The Japan Society of Applied Physics

\section{Introduction}

Semiconductor double quantum dots (DQDs) are promising candidates for charge qubits as basic information storage elements in quantum computer architectures. ${ }^{1)}$ Indeed, recent studies showed the possibility of implementing quantum operations in GaAs/AlGaAs-heterostructure-based DQDs. ${ }^{2,3)}$ In such an approach, DQDs operate as a two-level system where the two logical states correspond to an excess electron being localized in one dot or another. However, various mechanisms may destroy the coherence in the system since the DQDs can never be isolated completely from their environment. Within the possible decoherence mechanisms, the electron-phonon interaction is the process that has been investigated the most, both experimentally ${ }^{4}$ and theoretically. ${ }^{5-7)}$ Because of its intrinsic physical properties, particularly the absence of piezoelectric interaction and its compatibility with the silicon-based industry standards for complementary metal-oxide-semiconductor (CMOS) technology, silicon DQDs have drawn much attention and many experimental investigations have been conducted, recently. ${ }^{8-10)}$ Decoherence processes could also be minimized by tailoring the phonon density of states when reducing the degree of freedom of the phonon propagation within the nanostructures, as theoretically discussed in refs. 11-13. Thus, it is expected that a system composed of DQDs embedded in a suspended nanostructure (suspended DQDs, SDQDs) can substantially reduce the decoherence in charge qubits. ${ }^{13)}$ On the other hand, in spin-based qubits, the decoherence is expected to be smaller than that in the charge qubits ${ }^{14}$ ) because of the absence of intrinsic nuclear spin in natural silicon-28. ${ }^{15)}$ Processing a double-dot structure within a suspended nanostructure would give a significant advantage to spin-based qubit approaches because decoherence could be reduced further by manipulating the spin-orbit interaction. ${ }^{16,17)}$

Although recent technological development have enabled the realization of nanometer-scale suspended single quantum

*E-mail address: j.ogi@neo.pe.titech.ac.jp dots, most research has concentrated on studying singleelectron tunneling in silicon devices ${ }^{18)}$ or in GaAs devices. ${ }^{19,20)}$ Only few experimental investigations of phonon tailoring in SDQDs have been performed so far. ${ }^{21)}$ In particular, there is no investigation of electron-phonon interaction in silicon SDQDs.

In this paper, we present the first study of the electronphonon interaction in electron-beam (EB)-fabricated silicon SDQDs through the observation of inelastic tunneling in the drain-to-source current. Experimental results are discussed and well explained by the shape of the theoretically calculated phonon spectral density of states.

\section{Device Structure and Measurement Method}

The fabrication process of a suspended quantum dot has already been established in our previous work by combining EB lithography and thermal oxidation. ${ }^{18)}$ A thin-film channel was first patterned on a heavily doped silicon-on-insulator (SOI) wafer with a wedged shape on the film edge using EB lithography and electron-cyclotron-resonance reactive ion etching (ECR-RIE). The $\mathrm{SiO}_{2}$ layer under the film was etched out with hydrofluoric acid to form a suspended thin film and an SDQDs channel. Finally, SDQDs were thermally oxidized to passivate the device surface and reduce dot size.

A 40-nm-thick and 150-nm-wide silicon thin film was obtained and suspended over the silicon substrate [Figs. 1(a) and 1(b)]. The DQDs were embedded in the film by patterning the three constricted parts on the film edge. The constricted parts are expected to act as a tunnel barrier owing to the quantum size effect and thus to define coupled SDQDs. The sample surface was passivated and a 10-nmthick thermally oxidized layer was formed. Two side-gate electrodes control the electrochemical potentials of each quantum dot.

When the chemical potentials of each dot align, a single electron elastically tunnels through the potential barrier between the DQDs. On the other hand, when the chemical potentials do not align, an electron inelastically tunnels with the emission or the absorption of a phonon of the same energy as the potential difference. 

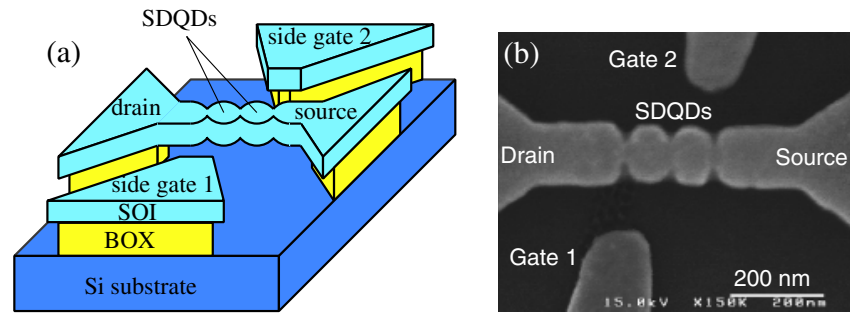

Fig. 1. (Color online) (a) Schematic three-dimensional view of the device and (b) SEM image of the fabricated SDQDs. SDQDs are located at the center of the suspended thin-film channel. The side-gate electrodes can modulate the chemical potentials in each quantum dot.

The inelastic tunnel rate $\Gamma_{\mathrm{C}}$ depends both on the phonon spectral density $J(\omega)$, where $\omega$ is the phonon frequency, and on the DQDs potential difference $\Delta E$ as expressed by ${ }^{4)}$

$$
\Gamma_{\mathrm{C}} \propto\left(\frac{T_{\mathrm{c}}}{\Delta E}\right)^{2} J(\Delta E) .
$$

The drain-to-source current $I_{\text {ine }}$ is then determined by the tunnel rates at each tunnel barrier as

$$
I_{\text {ine }}=e\left(\Gamma_{\mathrm{L}}^{-1}+\Gamma_{\mathrm{C}}^{-1}+\Gamma_{\mathrm{R}}^{-1}\right)^{-1},
$$

where $\Gamma_{\mathrm{L}}$ and $\Gamma_{\mathrm{R}}$ are respectively the left and right tunnel barrier rates.

In the region where $\Gamma_{\mathrm{C}}$ becomes smaller than $\Gamma_{\mathrm{L}}$ and $\Gamma_{\mathrm{R}}$, $I_{\text {ine }}$ is almost proportional to $\Gamma_{\mathrm{C}}$ and $J(\Delta E) / \Delta E^{2}$, so that a measure of inelastic current gives direct access to the phonon spectral density. In DQDs embedded in a suspended film, information about electron-phonon interaction is contained in phonon spectral density ${ }^{12,13)}$ and thus can be accessed via conductivity measurement.

\section{Measurement Results of the Single-Electron Transport via the SDQDs}

We observe the typical characteristics of a DQDs structure, e.g., two sets of charging lines at the crossing of which appears a pair of triangular regions where current is enhanced. The size of these regions also depends on the source-to-drain bias that is applied ${ }^{1)}$ [solid contour in Fig. 2(a) and the triangles in Fig. 2(b)]. However, for some range of gate voltages [dashed line contour in Fig. 2(a)], the DQDs characteristics are far more complicated and suggest the presence of an extra dot in the structure that may have been formed due to fluctuations in doping concentration. ${ }^{22)}$ In order to analyze the DQDs characteristics and inelastic current, we carefully chose one overlapped triangle, shown in Fig. 2(b), that is not affected by the charging line of the extra quantum dot.

The various capacitances of the DQDs structure could be estimated from the DQDs characteristics ${ }^{1)}$ and are shown in Table I. The sizes of the DQDs are calculated by iterative numerical calculations using the finite element method. Despite the symmetrical design of the constrictions, the dot closest to the drain electrode has a diameter of about $60 \mathrm{~nm}$, whereas the other dot is about $150 \mathrm{~nm}$ wide. This suggests that the larger dot has successfully been defined by realizing a constriction, but that the smaller dot has been induced by fluctuations in doping concentration in a constricted part.

As shown in Fig. 3, the current in the triangular region is well fitted by eight peaks whose dependence on the potential
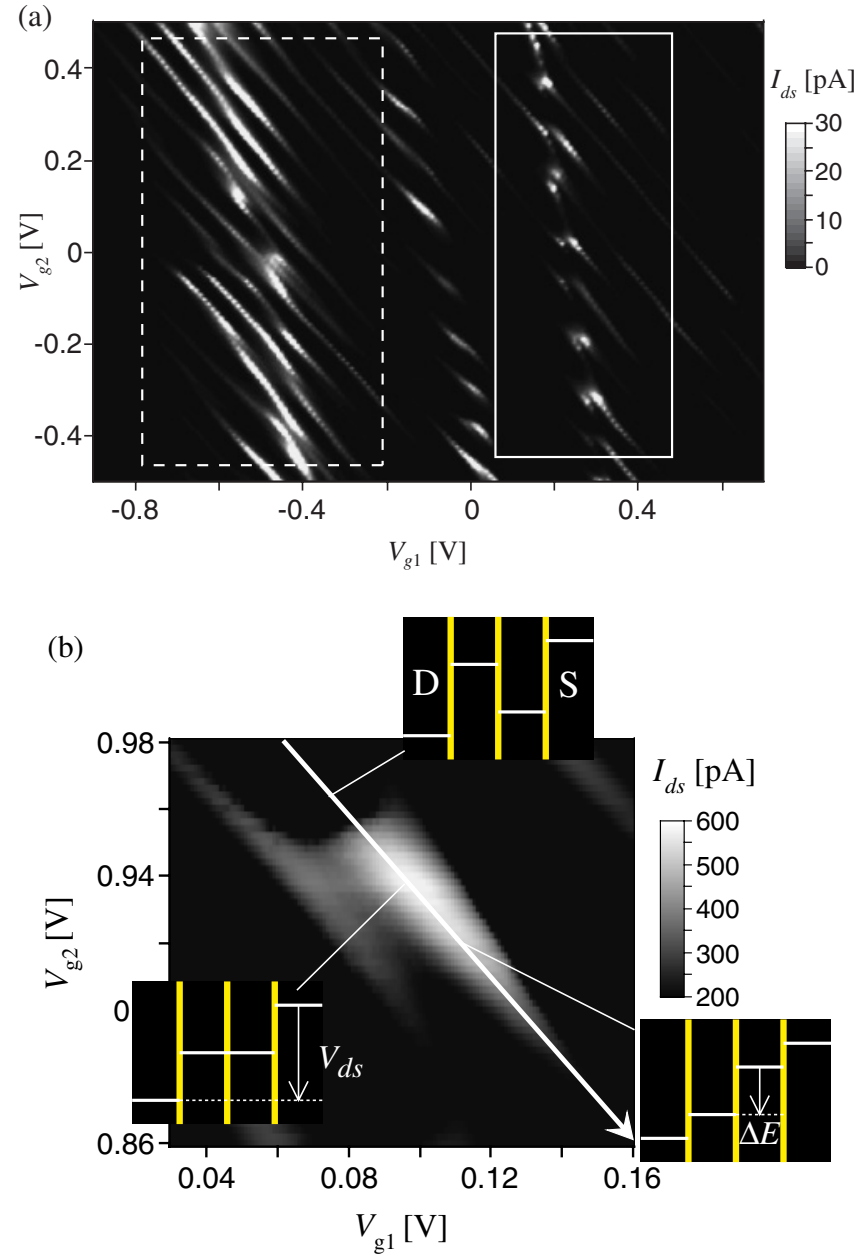

Fig. 2. (Color online) (a) Side gate voltage dependence of the drain-tosource current in SDQDs at $120 \mathrm{mK}$ with $V_{\mathrm{ds}}=200 \mu \mathrm{V}$. $V_{\mathrm{g} 1}$ and $V_{\mathrm{g} 2}$ are the voltages of the side gates 1 and 2 , respectively. The solid contour represents the range of gate voltages in which the structure behaves as DQDs, whereas the dashed contour shows a region sensitive to the extra dot. (b) Pair of overlapped triangles. The potential difference between the DQDs, $\triangle E$, can be detuned by sweeping the gate voltages along the white arrow. The insets schematically show the energy diagrams of the potential detuning at different gate voltages along the arrow direction, for a fixed positive source-to-drain bias $V_{\mathrm{ds}}$.

Table I. Values of the capacitances obtained experimentally. $C_{\mathrm{L}}, C_{\mathrm{m}}$, and $C_{\mathrm{R}}$ are the capacitances between the drain electrode and the left dot the left dot and the right dot, and the right dot and the source electrode, respectively. $C_{1(2) \mathrm{L}(\mathrm{R})}$ is the capacitance between side gate 1 (2) and the left (right) dot. The unit is aF.

\begin{tabular}{lrllllll}
\hline$C_{\mathrm{L}}$ & 81 & $C_{1 \mathrm{~L}}$ & 0.55 & $C_{2 \mathrm{~L}}$ & 0.15 & $C_{\mathrm{m}}$ & 32 \\
$C_{\mathrm{R}}$ & 330 & $C_{1 \mathrm{R}}$ & 1.6 & $C_{2 \mathrm{R}}$ & 1.6 & & \\
\hline
\end{tabular}

difference is given by $A \cosh ^{-2}\left[\log (3+2 \sqrt{2})\left(\Delta E-\Delta E_{0}\right) /\right.$ $2 k_{\mathrm{B}} T^{*}$, where $A, \Delta E_{0}$, and $T^{*}$ are fitting parameters. $T^{*}$ is depends not only on electron temperature in the case of inelastic tunneling, but also on the phonon dispersion relation. However, for elastic tunneling, $T^{*} \sim T_{\mathrm{e}}$ where $T_{\mathrm{e}}$ is the electron temperature. $T_{\mathrm{e}}$ could be obtained independently by measuring the temperature dependence of the peak width. Experimentally, $T_{\mathrm{e}} \sim 500 \mathrm{mK}$ although a small variation has been noticed from Coulomb peak to Coulomb peak. This corresponds to single-electron transport tunneling 

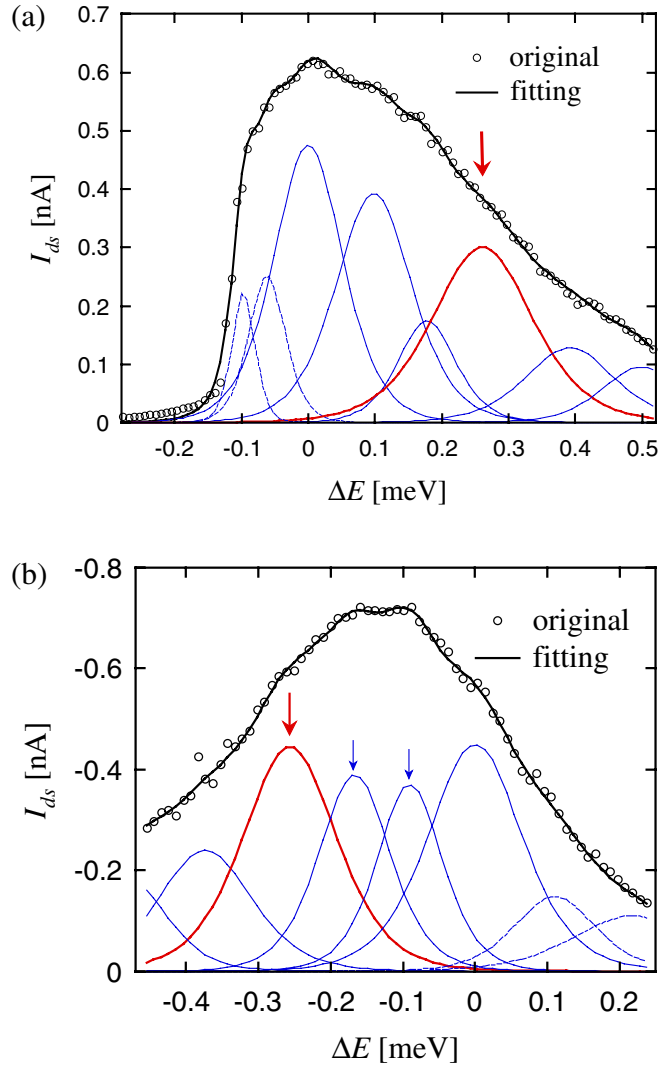

Fig. 3. (Color online) Drain-to-source current $I_{\text {ds }}$ versus the potential difference $\Delta E$ with (a) $V_{\text {ds }}=500 \mu \mathrm{V}$ and (b) $V_{\text {ds }}=-500 \mu \mathrm{V}$. The original data (circle) are fitted by a sum of peaks. The largest peak, as indicated by the large arrow, is always observed. The sign of the $\Delta E$ is defined as the one of $V_{\mathrm{ds}}$ as shown in the energy diagram in Fig. 2.

peaks that are thermally broadened. ${ }^{23)}$ The side-gate voltage was converted to $\Delta E$ on the horizontal axis using the bias dependence of the triangle size in Fig. 2. Note that a deviation of about $20 \mu \mathrm{eV}$ may arise from the conversion due to the obscure edges of the triangle. For positive $V_{\mathrm{ds}}$, the largest peak centered at $\Delta E=0$ corresponds to elastic tunneling. The peaks centered at $\Delta E>0$ correspond to inelastic tunneling and reflect the shape of the phonon spectral density of the SDQDs. For negative $V_{\mathrm{ds}}$, the peaks centered at $\Delta E<0$ correspond to inelastic tunneling. The small peaks centered at $\Delta E<0$ for positive $V_{\mathrm{ds}}$ and at $\Delta E>0$ for negative $V_{\mathrm{ds}}$ [the dashed lines in Figs. 3(a) and $3(\mathrm{~b})$ ] presumably correspond to cotunneling. It should be noted that the magnitude of these peaks associated with cotunneling is different in the spectra for the positive and negative $V_{\mathrm{ds}}$ because of the size asymmetry of the DQDs.

\section{Discussion}

The measured SDQDs structure was modeled using a suspended SOI film sandwiched by $\mathrm{SiO}_{2}$ layers and assuming a cylindrical structure for the quantum dots, as shown in Fig. 4(a). The model was similar to that used by Liao et $a l .{ }^{13)}$ for disklike DQDs embedded in a thin-film monolayer. However, our model takes into account the finite thickness of the cylindrical DQDs, the asymmetry of the DQDs, the multilayered films, and the finite width of the films. (a) Cylindrical DQDs
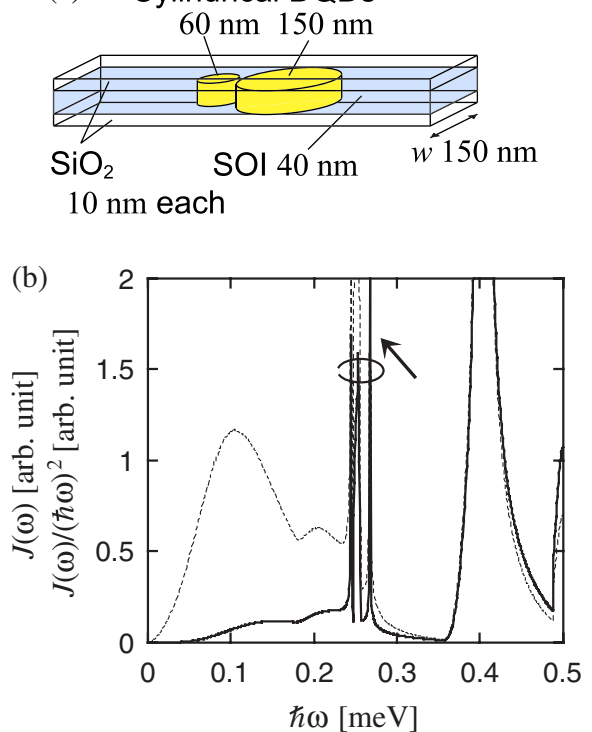

Fig. 4. (Color online) (a) Model used for the calculation of the phonon spectral density in the fabricated SDQDs device. Cylindrical DQDs with asymmetrical sizes are embedded in a 40-nm-thick SOI sandwiched by $10-\mathrm{nm}$-thick $\mathrm{SiO}_{2}$ layers. (b) Phonon spectral density theoretically obtained with the model (solid line). The dashed line represents the ratio of the phonon spectral density to the square of the phonon energy. The dashed line is normalized so that its maximum value corresponds to those of the solid line.

Spectral density was calculated by modeling the electronphonon interaction in disk shaped DQDs of zero thickness and then integrating over the thickness of the DQDs. Both dilatational and flexural modes are considered depending on the position of the DQDs along the film thickness. The different dot form factors are taken into account owing to the asymmetry in dot size. The relative thickness of the $\mathrm{SiO}_{2}$ layer covering the SOI layer, is renormalized in order to take into account the difference between the acoustic phonon velocities of $\mathrm{Si}$ and $\mathrm{SiO}_{2}$ so that the initial 10-nm-thick $\mathrm{SiO}_{2}$ layer is modeled by a 14-nm-thick silicon layer. Within such approximation, the film is approximated by a silicon film of $68 \mathrm{~nm}$ thickness. The longitudinal acoustic velocity $c_{1}$ and the transverse acoustic velocity $c_{\mathrm{t}}$ used for $\mathrm{Si}$ are 8430 and $5840 \mathrm{~m} / \mathrm{s}$, respectively. ${ }^{24)}$ Figure 4(b) shows the obtained phonon spectral density for the device.

By comparing the theoretical dependence of $J(\omega) /(\hbar \omega)^{2}$ on the phonon energy to the experimental dependence of drain-to-source current on DQDs potential energy difference, we observe similarities in the peaks positions. However, the small film width induces other effects that have not been taken into account in the theoretical calculation and that may explain the differences in peak heights and widths. Effectively, at such a small film width $w$, phonons are confined, causing phonon dissipation. ${ }^{12)}$ As a consequence, peaks become broadened by an energy $\hbar c_{1} / w \sim 40 \mu \mathrm{eV}$ with $w=150 \mathrm{~nm}$, which is inversely proportional to phonon lifetime for a width $w$.

The relatively large peak observed experimentally at $\Delta E=260 \mu \mathrm{eV}$ (Fig. 3) could be associated with the group of peaks that corresponds to the enhancement of the electron-phonon interaction, as seen in the theoretically calculated phonon spectral density. Broadening due to the 
electron temperature $(\sim 50 \mu \mathrm{eV})$ and the finite width of the film could result in the merging of the three peaks in Fig. 4 into a single broad peak. The other peaks observed at $\Delta E=100 \mu \mathrm{eV}, \Delta E=180 \mu \mathrm{eV}$, and $\Delta E=390 \mu \mathrm{eV}$ in the experimental results also agree well with the small broad peaks and the large broad peak in Fig. 4. In the case of inelastic tunneling, the current peak value depends on $J(\omega)$, the tunnel coupling $T_{\mathrm{c}}$ between the DQDs, as well as the tunnel rates $\Gamma_{\mathrm{L}}$ and $\Gamma_{\mathrm{R}}$ [eqs. (1) and (2)] so that the difference in peak height between the experiment and the theory could easily be attributed to the dependence on $\Gamma_{\mathrm{L}}$ and/or $\Gamma_{\mathrm{R}}$ as well as to the high electron temperature.

The comparison between the data obtained at positive and negative source-to-drain biases [respectively shown in Figs. 3(a) and 3(b)] discards the possibility that the observed peaks in the tunneling current are due to state quantization. For example, when $V_{\mathrm{ds}}<0$, the peaks observed at $\Delta E=$ $-260,-90,-170$, and $-370 \mu \mathrm{eV}$ are highly symmetric in position to those observed when $V_{\mathrm{ds}}>0$. This is consistent with the fact that the inelastic tunneling between the ground states has a symmetrical dependence on $V_{\mathrm{ds}}$ although the tunneling from the ground state to the excited state should show an asymmetrical dependence on $V_{\mathrm{ds}}$ due to the asymmetrical DQDs sizes. The small differences in peak shape and height observed upon a change in the sign of $V_{\mathrm{ds}}$ are presumably caused by excited states in the data obtained at negative biases. Because of the dot asymmetry, excited states in the large right dot have small energies and may appear at the negative $V_{\mathrm{ds}}$ leading to the presence of sharp peaks at $\Delta E=-90$ and $-170 \mu \mathrm{eV}$ as indicated by the small arrows in Fig. 3(b). These energies are almost consistent with the energy of the excited states in the 150-nm-wide and 40 -nm-thick quantum dot. The peaks at a positive $V_{\mathrm{ds}}$ are not affected by the excited states owing to their large energy in the smaller dot.

In addition, a large inelastic peak at $\Delta E=250 \mu \mathrm{eV}$ was also observed in another triangle at a different number of electrons, as shown in Fig. 5. This result again discards the possibility that this peak results from the tunneling through excited states because of the variation in excited state energy with number of electrons. This indeed supports the reproducibility of the results and the reliability of the peak fitting method that is used. As a consequence, it is likely that the peaks in the inelastic tunneling current, especially at approximately $\Delta E=260 \mu \mathrm{eV}$, result from electron-phonon interaction in SDQDs.

The enhanced interaction observed at approximately $\Delta E(=\hbar \omega)=260 \mu \mathrm{eV}$ is a specific feature of SDQDs. The elastic wave propagating in such a suspended thin film shows a nonlinear behavior and its group velocity could vanish at specific propagation mode energy. ${ }^{12)}$ Here, the enhanced peak correspond to the first excited dilatational mode, which shows a strong interaction with electrons confined in laterally configured SDQDs owing to the symmetrical deformation potential in the direction perpendicular to the film. The value of $260 \mu \mathrm{eV}$ is determined by the equivalent film thickness of $68 \mathrm{~nm}$ and acoustic velocity. The zero group velocity of the dilatational mode results in a strong electron-phonon interaction that was indeed observed experimentally. The larger acoustic velocities in silicon, and consequently the larger peak energies and separation may

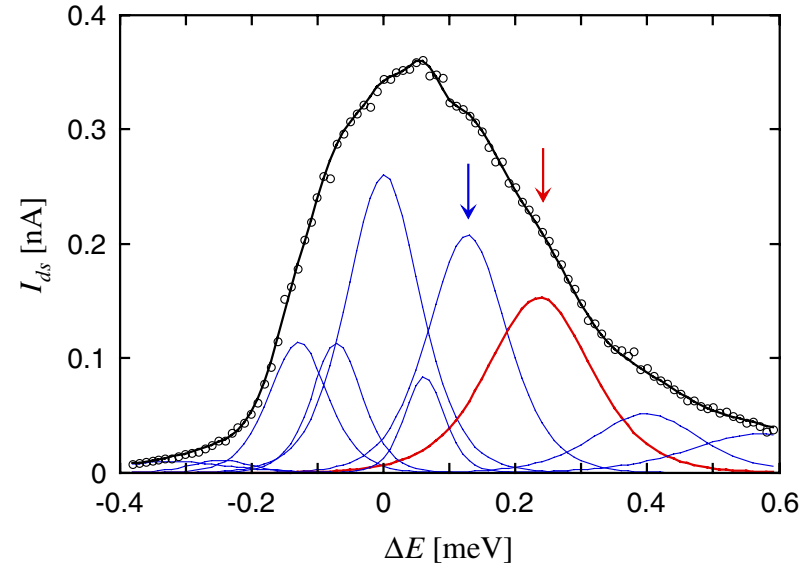

Fig. 5. (Color online) Drain-to-source current $I_{\text {ds }}$ versus the potential difference $\Delta E$ with $V_{\mathrm{ds}}=500 \mu \mathrm{V}$ in another triangle at a different number of electrons. The large peak centered at $\Delta E=250 \mu \mathrm{eV}$ was again observed. The large peak at approximately $\Delta E=130 \mu \mathrm{eV}$ corresponds to the charging line of the extra quantum dot and may suppress the observation of the peaks at $\Delta E=100 \mu \mathrm{eV}$ and $\Delta E=180 \mu \mathrm{eV}$ shown in Fig. 3(a).

explain why such an interaction could be observed in our device, but not in GaAs SDQDs. ${ }^{20)}$ The strength of the interaction depends on quantum dot size and DQDs positions, which are related to the wave number of the interacting phonons and the periodicity of the deformation potential, respectively. However, the interaction enhancement is determined exclusively by phonon density, and the effects of the size and position on the enhancement are not significant.

Together with peak broadening due to the small slab width, the overlap between the flexural mode and the dilatational phonon mode in cylindrical DQDs ${ }^{13}$ may be responsible for the electron-phonon interaction not being suppressed in our experiments. However, we did observe tunneling peaks that are related to the various branches in the phonon dispersion ${ }^{11}$ and distinctive phonon modes (the dilatational and flexural modes). ${ }^{13)}$ These results as well as the specific properties of silicon provide significant advantage towards the realization of engineered Si SDQDs in which the electron-phonon interaction could be suppressed. For example, the use of a thinner film may increase specific phonon energy and consequently decrease the extent of relative peak broadening, or the use of cylindrical nanowires could decrease the absolute peak broadening by reducing the phonon-energy dissipation due to its symmetric shape. Also, ultrathin SOI could potentially suppress the electron distribution in the middle plane of the slab and reduce the overlapping of the flexural mode with the dilatational mode.

Although these devices were realized with the aim of realizing a quantum information processing device, the observation of the enhancement of the electron-phonon interaction holds promise for other applications. Extremely small mass detectors are potentially realizable because phonon modes, which are sensitive to atomic-scale mass changes, can be detected via electron transport measurement. In addition, in such a regime where electrons interact strongly with phonons, detectors of microscopic mechanical motion $^{25,26)}$ or cooling of quantized phonon modes ${ }^{27)}$ could also be realized. 


\section{Conclusions}

We have successfully fabricated silicon SDQDs by using EB lithography and thermal oxidation in which clear singleelectron transport properties could be observed. The large inelastic tunneling obtained, the electrons are transferred from one dot to another, is well explained by the model of electron-phonon interaction in a suspended thin film. The strong electron-phonon interaction is caused by the presence of a zero group velocity in the phonon dispersion relations of SDQDs. These results suggest the existence of distinctive phonon modes in silicon SDQDs and the possibility of tailoring of the electron-phonon interaction in such a structure. $^{28)}$

\section{Acknowledgements}

The device was fabricated using the EB-lithography facilities in Quantum Nanoelectronics Research Center, Tokyo Institute of Technology, Japan. This work was partly supported by a Grant-in-Aid for Scientific Research (No. 19206035) from the Japan Society for the Promotion of Science and a Grant-in-Aid for Scientific Research (No. 2170137) from the Ministry of Education, Culture, Sports, Science and Technology, Japan (MEXT), Global COE Program, "Photonics Integration-Core Electronics", MEXT, and Special Coordination Funds for Promoting Science and Technology in Japan.

1) W. G. van der Wiel, S. D. Franceschi, J. M. Elzerman, T. Fujisawa, S. Tarucha, and L. P. Kouwenhoven: Rev. Mod. Phys. 75 (2003) 1.

2) T. Hayashi, T. Fujisawa, H. D. Cheong, Y. H. Jeong, and Y. Hirayama: Phys. Rev. Lett. 91 (2003) 226804.

3) J. R. Petta, A. C. Johnson, C. M. Marcus, M. P. Hanson, and A. C. Gossard: Phys. Rev. Lett. 93 (2004) 186802.

4) T. Fujisawa, T. H. Oosterkamp, W. G. van der Wiel, B. W. Broer, R. Aguado, S. Tarucha, and L. P. Kouwenhoven: Science 282 (1998) 932.

5) Z. J. Wu, K. D. Zhu, X. Z. Yuan, Y. W. Jiang, and H. Zheng: Phys. Rev. B 71 (2005) 205323.
6) S. Vorojtsov, E. R. Mucciolo, and H. U. Baranger: Phys. Rev. B 71 (2005) 205322.

7) M. J. Storcz, U. Hartmann, S. Kohler, and F. K. Wilhelm: Phys. Rev. B 72 (2005) 235321.

8) J. Gorman, D. G. Hasko, and D. A. Williams: Phys. Rev. Lett. 95 (2005) 090502.

9) H. Liu, T. Fujisawa, H. Inokawa, Y. Ono, A. Fujiwara, and Y. Hirayama: Appl. Phys. Lett. 92 (2008) 222104.

10) W. H. Lim, H. Huebl, L. H. Willems van Beveren, S. Rubanov, P. G. Spizzirri, S. J. Angus, R. G. Clark, and A. S. Dzurak: Appl. Phys. Lett. 94 (2009) 173502.

11) N. Bannov, V. Aristov, V. Mitin, and M. A. Storscio: Phys. Rev. B 51 (1995) 9930

12) S. Debald, T. Brandes, and B. Kramer: Phys. Rev. B 66 (2002) 041301(R).

13) Y. Y. Liao, Y. N. Chen, W. C. Chou, and D. S. Chuu: Phys. Rev. B 77 (2008) 033303.

14) A. C. Johnson, J. R. Petta, J. M. Taylor, A. Yacoby, M. D. Lukin, C. M. Marcus, M. P. Hanson, and A. C. Gossrad: Nature 435 (2005) 925.

15) A. Morello, C. C. Escott, H. Huebl, L. H. Willems van Beveren, L. C. L. Hollenberg, D. N. Jamieson, A. Z. Dzurak, and R. G. Clark: Phys. Rev. B 80 (2009) 081307(R)

16) W. Pan, X. Z. Yu, and W. Z. Shen: Appl. Phys. Lett. 95 (2009) 013103

17) Y. Y. Liao, Y. N. Chen, D. S. Chuu, and T. Brandes: Phys. Rev. B 73 (2006) 085310.

18) J. Ogi, Y. Tsuchiya, S. Oda, and H. Mizuta: Microelectron. Eng. 85 (2008) 1410.

19) S. J. Chorley, C. G. Smith, F. Perez-Martinez, J. Prance, P. Atkinson, D. A. Ritchie, and G. A. C. Jones: Microelectron. J. 39 (2008) 314.

20) C. Rossler, M. Bichler, D. Schuh, W. Wegscheider, and S. Ludwig: Nanotechnology 19 (2008) 165201.

21) E. M. Weig, R. H. Blick, T. Brandes, J. Kirschbaum, W. Wegscheider, M. Bichler, and J. P. Kotthaus: Phys. Rev. Lett. 92 (2004) 046804.

22) G. J. Evans, H. Mizuta, and H. Ahmed: Jpn. J. Appl. Phys. 40 (2001) 5837.

23) C. W. J. Beenakker: Phys. Rev. B 44 (1991) 1646.

24) V. A. Fonoberov and A. A. Balandin: Nano Lett. 6 (2006) 2442.

25) H. Park, J. Park, A. K. L. Lim, E. H. Anderson, A. P. Alicisatos, and P. L. McEuen: Nature 407 (2000) 57.

26) B. J. LeRoy, S. G. Lemay, J. Kong, and C. Dekker: Nature 432 (2004) 371.

27) P. J. Koppinen and I. J. Maasilta: Phys. Rev. Lett. 102 (2009) 165502.

28) After acceptance of our paper, a related study of probing confined phonon modes in a nonsuspended InAs nanowire double quantum dots was reported in C. Weber, A. Fuhrer, C. Fasth, G. Lindwall, L. Samuelson, and A. Wacker: Phys. Rev. Lett. 104 (2010) 036801 